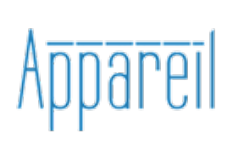

\title{
Appareil
}

4 | 2010

Biopolitique, éthique et subjectivation, questions de modernité

\section{Les cours enregistrés de Gilles Deleuze à l'université de Paris 8, 1979-1987}

Une inscription de l'événement Mai 68

\section{Frédéric Astier}

\section{(2) OpenEdition \\ Journals}

Édition électronique

URL : https://journals.openedition.org/appareil/896

DOI : 10.4000/appareil.896

ISSN : 2101-0714

Éditeur

MSH Paris Nord

\section{Référence électronique}

Frédéric Astier, «Les cours enregistrés de Gilles Deleuze à l'université de Paris 8, 1979-1987 », Appareil [En ligne], 4 | 2010, mis en ligne le 27 janvier 2010, consulté le 12 octobre 2021. URL : http:// journals.openedition.org/appareil/896; DOI : https://doi.org/10.4000/appareil.896

Ce document a été généré automatiquement le 12 octobre 2021.

\section{(†)

Appareil est mis à disposition selon les termes de la Licence Creative Commons Attribution - Pas d'Utilisation Commerciale - Pas de Modification 4.0 International. 


\title{
Les cours enregistrés de Gilles Deleuze à l'université de Paris 8, 1979-1987
}

\author{
Une inscription de l'événement Mai 68
}

\author{
Frédéric Astier
}

1 Les archives sonores des cours de Gilles Deleuze à l'université de Paris 8, 1979-1987 (177 cours, 400 heures) nous transportent, auditeurs et lecteurs, entre les flux et reflux de paroles et les lignes des chapitres du philosophe. Le cours était le moment d'élaborer ou de perfectionner un concept, d'abord à partir d'une problématique abstraite, puis d'exemples concrets qui s'adressaient tant aux spécialistes des idées qu'aux «non philosophes ». Un énoncé philosophique se répète d'un cours à l'autre et s'enrichit d'une multiplicité de références artistiques, politiques et scientifiques; une anecdote agence un mode de vie personnel et une approche conceptuelle. Ces différents points de vue élaborent une expérimentation. Dans un même mouvement, un nouveau régime de la parole philosophique et une "pédagogie » s'inventent. Éloigné du discours de la philosophie académique, l'enseignement de Gilles Deleuze à Vincennes fait événement, sur un mode impersonnel (la voix), produit une rencontre inédite, inaugure une pensée de la création et du virtuel. Sa philosophie orale constitue le préalable de ses livres et un laboratoire nécessaire pour un "affect du concept», selon un style dialogique. Elle se poursuit aujourd'hui en direction d'un « devenir auditeur » qui renoue ainsi avec la tradition orale, l'écriture créative, la transmission des notions philosophiques et la recherche universitaire vivante.

2 Traverser la rue de Richelieu par les souterrains de la Bibliothèque nationale pour acheminer d'antiques disques 78 tour/mn vers les studios Vivienne du département de la Phonothèque, ce chemin m'était familier. Mais un matin, surprise, derrière un épais mur de verre, j'entendis la voix de Gilles Deleuze, des phrases sur un bruit de fond d'autoroute, amplifié par une salle préfabriquée habituellement destinée aux migrants étrangers (les LOPOFA). Des cassettes audio de quelques étudiants nous étaient parvenues en très grande quantité. Quelques mois plus tard, humble employé, fort de 
quelques années d'études à l'université de Paris 8 , je proposais à mes responsables de m'occuper du pointage, et par suite du classement, de l'étiquetage, de la cotation et de la vérification dite technique des durées de ces enregistrements transférés sur disques compacts, et pour cela, il fallut les entendre.

Hasard, je me souviens avoir ri en l'écoutant, lorsque de sa voix rauque et caillouteuse, il affirme que le philosophe doit parler au non philosophe, ou encore, que l'idiot de Nicolas de Cuses est un très bon observateur. Je me sentais dans la peau de l'un et de l'autre, c'est peu dire. Et c'est ainsi que des mois durant, je pris note des idées développées dans ces 177 cours, 400 heures - de 1979 à 1987, un travail d'ombre.

4 Aborder la pensée de Gilles Deleuze, pensais-je, nécessitait des rudiments et un horizon de références, car notre auteur avait beau dire «ne vous inquiétez pas, tout le monde peut comprendre ", philosophe ou non, je ne le croyais guère. Son invite cependant fut très efficace - «prenez ce que vous voulez, ce qui vous convient, c'est vous qui voyez »-, elle me permit d'être à l'aise, un certain confort et en même temps, l'obligation de se demander : mais qu'est-ce qui me concerne dans ce qu'il dit?

5 Ce qui me convient, ce qui me parle, ce qui me fait penser à quelque chose, le propos est déculpabilisant et incite à travailler pour soi-même et par soi-même. Deleuze sait instituer l'autre comme auteur : de ses notes, ses connexions, ses rapprochements, ses propres plans.

6 Puis, après avoir laissé reposer mon appareil auditif, une année sans doute, je repris l'écoute des cours sur certaines années afin de vérifier plus calmement mes prises de notes, sur quelques séances, durant lesquelles j'avais dû certainement somnoler, notamment la quatrième et dernière année consacrée par Deleuze au cinéma.

7 Les cours enregistrés de Gilles Deleuze, au lendemain du transfert de l'université de Paris 8 de Vincennes à Saint-Denis, sont consultables à la Bibliothèque nationale de France, à la Bibliothèque universitaire de Paris 8 , et le sont progressivement sur le site La voix de Gilles Deleuze en ligne, université de Paris 8.

8 Le style Deleuze. On peut dire que ses cours lui permettaient d'expérimenter des suites logiques, celles de ses futurs chapitres, mais à une vitesse inversée du brillant professeur. Très lentement, par flux et reflux, il avançait, reculait. Deleuze manifestement suivait des notes, très précises, même si ses références d'éditions, de dates, ou de pages étaient lacunaires. Son cours était expérimentation au sens fort. Il entendait, si les connexions se réalisaient de temps à autre, les petites lumières des regards.

9 Quelques lignes de force traversent les cours de Gilles Deleuze. Tout d'abord, sa minutie. L'air de rien, la préparation est extrême, tous les axes sont écrits, mais les développements se réalisent par vague, par répétitions préalables. C'est là toute la dimension construite du cours, sa maîtrise et son unité. Pas de place pour les charmes de l'orateur, ni d'effet de manche, c'est l'auditeur qui perçoit, qui voit, qui évalue ses besoins.

10 Vient ensuite la trame des cours, la durée de la pensée laborieuse et ses reprises, les notions abstraites et les enchaînements que Deleuze vérifie. Il s'oblige à préciser, à lever les malentendus, à refaire des ponts pour la compréhension. Comme on le dit d'un médicament, il parle avec des effets retardés, il reprend un nœud noué bien avant, il reformule autrement et fait un point, et il rassure : « sentez comme c'est beau, laissezvous aller, vous comprendrez plus tard ». 
11 Enfin, un dernier étage, pour les instruits en philosophie, pour lesquels il exige qu'ils aient absolument compris, Deleuze a besoin d'eux, il en va de la possibilité du cours prochain, donc de sa préparation, de sa répétition. Ce pourquoi, parfois encore, Deleuze considère que sa séance a échoué dans la compréhension, a mené à une impasse, ce qu'il ne manque pas d'affirmer au cours suivant; et donc il recommence, reprendre le cours précédent.

12 L'auditeur et le lecteur ne s'en étonneront pas. Les cours enregistrés et leurs transcriptions n'élaborent pas un programme de philosophie, un index sûr ni un sommaire, ils sont au mieux une distribution aléatoire de concepts, d'auteurs, de phrases au fil des séances, des mois et des années durant. Plutôt que de chercher à couvrir tous les ensembles conceptuels, Deleuze incite à naviguer en nomade, à la recherche de ce qui nous parle le plus. Ses cours sont à suivre comme un pêcheur au bord d'un fleuve qui suit son bouchon, il faut être patient, accepter de ne pas comprendre, accepter aussi ce régime d'hésitation qui, finalement, autorise l'auditoire à le suivre malgré sa méconnaissance.

13 Un dernier mot sur le net refus de Deleuze de laisser publier ses cours. C'est que pour lui, parler n'est pas écrire. Entre les deux, il y a une rupture radicale. Si le parler peut supporter un régime d'hésitation, des suspensions et des béances, des rires et des cris, l'écriture est une opération de refroidissement. Le parler n'a pas à être écrit, ni à être retranscrit donc, car il est "peuplé de voix », impersonnel, il met en mouvement des bandes, ramasse, capte et bégaie.

14 Comme un ensemble d'anneaux brisés, parler est souvent fait de pourparlers, histoire de dire qu'on avance, juste un bref moment en interaction qui doit rester comme tel.

15 Parions que les cours enregistrés rendent quelques perspectives plus visibles, éclairent les problèmes posés par tel peintre, tel cinéaste, permette de rejoindre des concepts en fonction de tel problème philosophique, artistique, politique, personnel. Parions aussi que durant l'écoute, vous saurez perdre votre temps, afin de gagner en vitesse de pensée. 